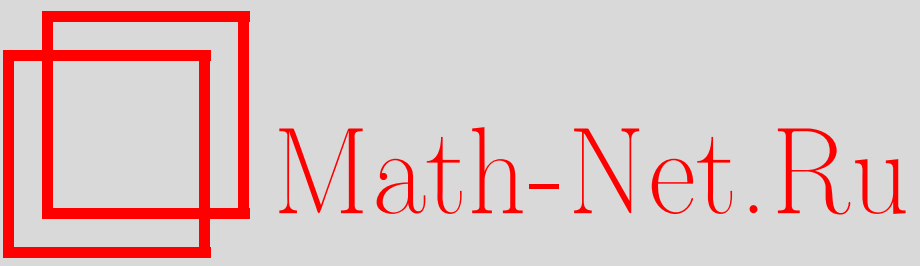

А. А. Русаков, Предельная теорема для числа выходов за высокий уровень огибающей гауссовского стационарного случайного процесса, УМH, 2003, том 58, выпуск 6, 163-164

DOI: https://doi.org/10.4213/rm688

Использование Общероссийского математического портала Math-Net.Ru подразумевает, что вы прочитали и согласны с пользовательским соглашением

http://www.mathnet.ru/rus/agreement

Параметры загрузки:

IP: 18.234 .156 .22

26 апреля 2023 г., 10:47:41 


\title{
ПРЕДЕЛЬНАЯ ТЕОРЕМА ДЛЯ ЧИСЛА ВЫХОДОВ ЗА ВЫСОКИЙ УРОВЕНЬ ОГИБАЮЩЕЙ ГАУССОВСКОГО СТАЦИОНАРНОГО СЛУЧАЙНОГО ПРОЦЕССА
}

\author{
A. A. РуСАКОВ
}

Пусть $\boldsymbol{\xi}_{t}=\left(\xi_{1 t}, \xi_{2 t}\right)^{T}$ - двумерный вещественньй стационарный гауссовский случайньй процесс с нулевым средним. Запишем спектральное представление его первой компоненты в виде, [1],

$$
\xi_{1 t}=\int_{0}^{+\infty} \cos \lambda t d u(\lambda)+\int_{0}^{+\infty} \sin \lambda t d v(\lambda),
$$

где $u(\lambda), v(\lambda)$ - гауссовские случайные процессы с ортогональными приращениями, некоррелированные между собой, $\mathrm{E}|d u(\lambda)|^{2}=\mathrm{E}|d v(\lambda)|^{2}=(d F(\lambda)+d F(-\lambda)) / 2, F(\lambda)$ - спектральная функция случайного процесса $\xi_{1 t}$.

Пусть вторая компонента $\xi_{2 t}$ есть преобразование Гильберта процесса $\xi_{1 t}$ :

$$
\xi_{2 t}=\int_{0}^{+\infty} \sin \lambda t d u(\lambda)-\int_{0}^{+\infty} \cos \lambda t d v(\lambda) .
$$

Далее, обозначим $\lambda_{k}=\int_{-\infty}^{+\infty} \lambda^{k} d F(\lambda)-k$-й спектральньй момент стационарного процесса $\xi_{1 t}$. Ковариационная функция векторного процесса $\xi_{t}$ имеет вид

$$
\mathrm{E} \boldsymbol{\xi}_{t} \boldsymbol{\xi}_{t+\tau}^{T}=\left(\begin{array}{cc}
r_{11}(\tau) & r_{12}(\tau) \\
-r_{12}(\tau) & r_{11}(\tau)
\end{array}\right)
$$

где

$$
r_{11}(t)=\int_{0}^{+\infty} \cos \lambda t d F(\lambda), \quad r_{12}(t)=\int_{0}^{+\infty} \sin \lambda t d F(\lambda) .
$$

Не нарушая общности, будем предполагать, что $\lambda_{0}=r_{11}(0)=1$. Огибающей случайного процесса $\xi_{1 t}$ назьвают случайный процесс $\mathscr{E}_{t}=\sqrt{\xi_{1 t}^{2}+\xi_{2 t}^{2}},[1]$.

Пусть $N_{u}^{+}(\cdot)$ - точечный процесс выходов огибающей $\mathscr{E}_{t}$ за уровень $u$. Пусть $D_{u}-$ круг радиуса $u$, а $S_{u}$ - его граница, $S_{u}=\left\{\left(x_{1}, x_{2}\right) \in \mathbb{R}^{2}: x_{1}^{2}+x_{2}^{2}=u^{2}\right\}$. Пусть $N_{S_{u}}^{+}(\cdot)$ - точечньй процесс выходов векторного случайного процесса $\xi_{t}$ из круга $D_{u}$ за границу $S_{u}$. Выброс огибающей $\mathscr{E}_{t}$ за уровень $u$ соответствует выходу векторного процесса $\boldsymbol{\xi}_{t}$ за границу $S_{u}$ круга $D_{u}$. Следовательно, выходы огибающей $\mathscr{E}_{t}$ за уровень $u$ и выходы случайного процесса $\boldsymbol{\xi}$ из круга $D_{u}$ порождают один к тот же точечньй процесс на временной оси,

$$
N_{u}^{+}(\cdot)=N_{S_{u}}^{+}(\cdot)
$$

$N_{u}^{+}(\cdot)$ и $N_{S_{u}}^{+}(\cdot)$ определены на борелевских множествах из $[0, \infty)$. Справедлива формула для среднего числа выходов, [1], $\mathrm{E} N_{u}^{+}(J)=|J| \mu_{u}^{+}$, где $|J|$ - длина интервала $J$,

$$
\mu_{u}^{+}=u \sqrt{\frac{\lambda_{2}-\lambda_{1}^{2}}{2 \pi}} e^{-\frac{u^{2}}{2}}
$$

- интенсивность точечного процесса $N_{u}^{+}(\cdot)$.

Везде ниже предполагается конечность второго спектрального момента, $\lambda_{2}<\infty$. Отсюда в частности следует, что все пересечения окружности $S_{u}$ являются п.н. либо входами, либо выходами, см. [1]. 
Теорема 1. Пусть спектральная функция $F(\lambda)$ содержит ненулевую абсолютно непрерывную составляющую в своем разложении Лебега и для некоторых $\delta_{1}, \delta>0$ выполнены следующие условия:

$$
\begin{gathered}
\left|\dot{r}_{11}(0)-\dot{r}_{11}(t)\right|<|\ln | t||^{-(1+\delta)}, \quad|t|<\delta_{1}, \\
\lim _{t \rightarrow \infty}(\ln t) \sqrt{r_{11}^{2}(t)+r_{12}^{2}(t)}=0 .
\end{gathered}
$$

Тогда пересечения уровня и огибающей ஜ̈ стационарного гауссовского случайного прочесса $\xi_{1 t}$ в масштабе времени с единицей $\left(\mu_{u}^{+}\right)^{-1}$ образуют точечные прочессы, сходящиеся по распределению при $и \uparrow \infty к$ пуассоновскому точечному процессу, имеющему единичную интенсивность.

Доказательство теоремы основано на переходе к дискретному времени и использовании свойств перемешивания квантованного гауссовского процесса с дискретньм временем, аналогично [2], см. также [3]. При этом мы предварителњно изучаем свойства аппроксимации первого и второго моментов числа пересечений соответствующими функционалами в дискретном времени.

Рассмотрим последовательность значений векторного случайного процесса $\boldsymbol{\xi}_{t}$

$$
\left\{\boldsymbol{\xi}_{k h}\right\}, \quad k \in \mathbb{Z}=\{0,1,2, \ldots\},
$$

и порождаемый ею квантованньй точечный процесс $N_{u}^{+}(\cdot, h)$. Рассмотрим точечный процесс $N_{u}^{+}(\cdot, h)$ выходов последовательности $\left|\xi_{k h}\right|$ за уровень $u$. Имеем:

$$
\mathrm{E} N_{u}^{+}([k h,(k+1) h], h)=\mathrm{P}\left\{\boldsymbol{\xi}_{k h} \in D_{u} ; \boldsymbol{\xi}_{(k+1) h} \notin D u\right\}=\mathrm{P}\left\{\boldsymbol{\xi}_{k h}<u<\boldsymbol{\xi}_{(k+1) h}\right\} .
$$

Интенсивность точечного процесса $N_{u}^{+}(\cdot, h)$ будем обозначать $\mu_{u}^{+}(h)$, тогда

$$
\mu_{u}^{+}(h)=\frac{1}{h} P\left\{\boldsymbol{\xi}_{k h} \in D_{u} ; \boldsymbol{\xi}_{(k+1) h} \notin D_{u}\right\}, \quad \mathrm{E} N_{u}^{+}([0, k h], h)=\mu_{u}^{+}(h) k .
$$

Обозначим через $J_{(2)}(t)=\mathrm{E} N_{S_{u}}^{+}(J)\left(N_{S_{u}}^{+}(J)-1\right)$ второй факториальньй момент $N_{S_{u}}^{+}(\cdot)$ на интервале $J$ длины $t$.

Ниже приведены две леммы из трех, на которых основано доказательство теоремы. Из утверждения леммы 1 следует, сколь малшм нужно выбирать шаг квантования $h$, чтобы переходк квантованному процессу был корректен, т.е. чтобы $\mu_{u}^{+}(h) \sim \mu_{u}^{+}$при $u \uparrow \infty$. Из оценки второго факториального момента в лемме 2 следует асимптотическая ординарность рассматриваемого точечного процесса $N_{u}^{+}(\cdot)$. С помощью утверждения леммы 2 оцениваются вероятности $\mathrm{P}\left\{N_{u}^{+}(\cdot)=0\right\}$ и $\mathrm{P}\left\{N_{u}^{+}(\cdot)=1\right\}$. Третья лемма, которая здесь не приводится, является обобщением неравенства Бермана, см. [2]. Из нее следует асимптотическая независимость числа пересечений на непересекающихся интервалах. Пусть $w=w(u)$ - положительная функция такая, что $w \uparrow \infty$ при $u \uparrow \infty$.

Лемма 1. Пусть $\lambda_{2}<\infty$ и шаг квантования $h=1 /(u w)$, тогда $\mathrm{P}\left\{\boldsymbol{\xi}_{t} \in D_{u} ; \boldsymbol{\xi}_{t+h} \notin\right.$ $\left.D_{u}\right\} \sim \mu_{u}^{+}(u) h, \kappa о г \partial а u \uparrow \infty$.

Пусть $T=1 / \mu_{u}^{+}$, где $\mu_{u}^{+}$определено в $(2)$, а $s(u)$ - положительная возрастающая функция, $\frac{s(u)}{u} \uparrow \infty, \ln s(u)=o\left(u^{2}\right)$ при $u \uparrow \infty$.

ЛЕмма 2. Пусть случайный процесс $\boldsymbol{\xi}(t)$ удовлетворяет условиям теоремы 1. Тогда $J_{(2)}(T / s(u))=o(1 / s(u))$ npu $u \uparrow \infty$.

\section{СПИСОК ЛИТЕРАТУРЫ}

[1] Г. Крамер, М. Лидбеттер. Стационарные случайные процессы. М.: Мир, 1969. [2] S. M. Berman // Ann. Math. Statist. 1964. V. 35. № 2. P. 502-516. [3] V. I. Piterbarg. Asymptotic Methods in the Theory of Gaussian Processes and Fields. Providence, RI: Amer. Math. Soc., 1996. (Transl. Math. Monogr. V. 148.)

Московский государственньй

Принято редколлегией университет им. М.В. Ломоносова 23.09 .2003 